\title{
MODELING OF THE DAMAGED STATE \\ BY THE FINITE-ELEMENT METHOD \\ ON SIMULTANEOUS ACTION OF CONTACT AND NONCONTACT LOADS
}

\author{
S. S. Shcherbakov
}

UDC 539.3

A comparative analysis of the results of finite-element modeling of the damaged state of solid bodies in the vicinity of their contact interaction with simultaneous stretching or compression of one of the elements of the contact pair is presented. The values of dangerous volumes of these bodies were calculated by summing the volumes of their finite elements in which the average value of the intensity of stresses exceeds the limit of contact fatigue.

Keywords: deformed solid body, dangerous volume, stressed-strained state, finite-element method.

Introduction. In considering a diversity of practically important problems, for example, interaction of gearwheels, a wheel and a rail, and other objects, it becomes necessary to carry out a complex analysis of both the surface deformation with damage of these bodies in the local region of their contact, and volume deformation with damage of bodies from the bending load experienced by them.

Generally, the investigation of the damaged state of the indicated tribologically reliable systems during the interaction of their elements is based on the statistical model of a strained solid body having a dangerous volume [1]. Within the framework of this model, dangerous volumes are represented by three-dimensional regions where stresses attain the damaging level, i.e., the lower boundary of the fatigue limit scattering.

Model of a Deformable Solid Body Having a Dangerous Volume. The procedure of calculation of dangerous volumes of a tribosystem presupposes the knowledge of both the three-dimensional stressed-strained state of its elements caused by a local contact and three-dimensional loading, and of the limiting state of the system [2, 3].

In a tribosystem, in contrast, for example, to a shaft undergoing bending, all six independent components of the stress tensor are nonzero [4]. In the general case, the limiting state (formation of microcracks) at a certain point of the system can be attributable to several different components. Thus, the fatigue limit for a force system is determined from each component of the stress tensor as an extreme value of its distribution on action of the limiting loading.

Since dangerous volumes are a measure of the damaged state of deformed bodies, then, while analyzing them, one can point to specific regions (zones) in which the origination and development of both surface and internal cracks are possible. It is evident that the action of irreversible damages is implemented at the places where the corresponding dangerous volumes intersect (i.e., superimpose) or coincide.

We will consider the tensor of the mechanical parameter $\varphi_{i j}$ whose concrete representatives are the stress, $\sigma_{i j}$, and strain, $\varepsilon_{i j}$, tensors. For a homogeneous isotropic deformable solid body we determine the limiting normal and tangent values of $\varphi_{n}^{(* \lim )}$ and $\varphi_{\tau}^{(* \lim )}$ of the tensor $\varphi_{i j}^{( \pm * \lim )}$, as well as its limiting principal value $\varphi_{1}^{(* \lim )}$ and the limiting intensity $\varphi_{\text {int }}^{(* \lim )}$ :

$$
\begin{gathered}
\varphi_{\mathrm{n}}^{(* \lim )}=\max _{d V, i}\left(\left|\varphi_{i i}\left(F_{* \lim }, d V\right)\right|\right), \quad i=x, y, z, \\
\varphi_{\tau}^{(* \lim )}=\max _{d V, i, j}\left(\left|\varphi_{i j}\left(F_{* \lim }, d V\right)\right|\right), \quad i, j=x, y, z, \quad i \neq j,
\end{gathered}
$$

Belarusian State University, 4 Nezavisimost' Ave., Minsk, 220033, Belarus; email: sersher@tut.by. Translated from Inzhenerno-Fizicheskii Zhurnal, Vol. 85, No. 2, pp. 437-441, March-April, 2012. Original article submitted May 23, 2011. 

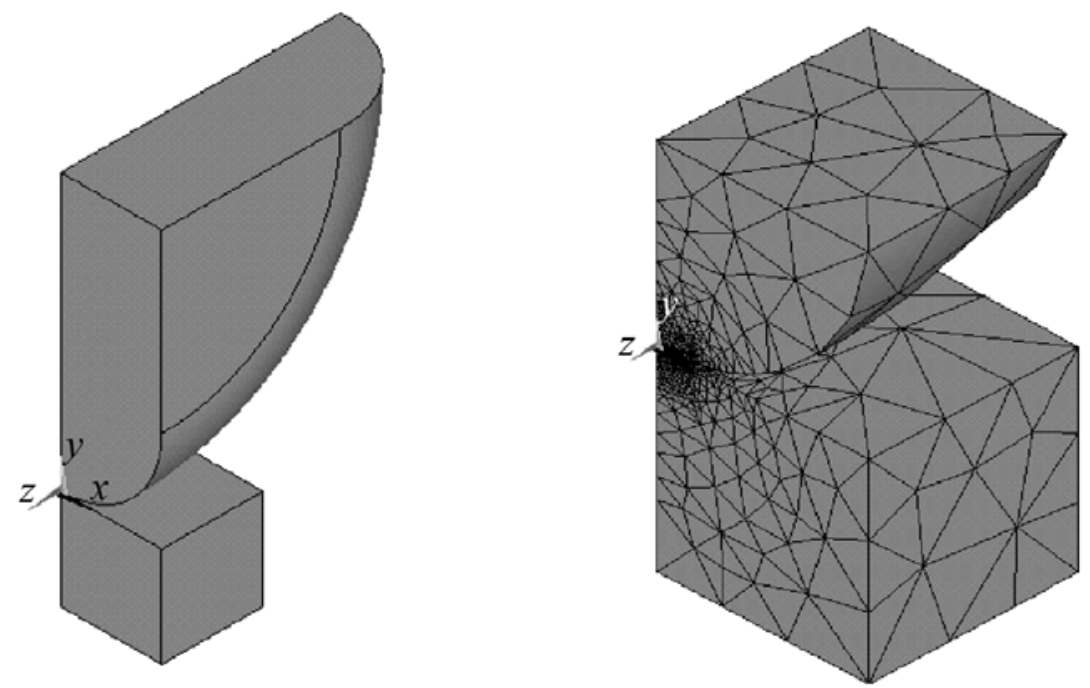

Fig. 1. Computational scheme and finite-element division of the indenter/elastic half-space system.

$$
\varphi_{1}^{(* \lim )}=\max _{d V}\left(\left|\varphi_{1}\left(F_{* \lim }, d V\right)\right|\right), \quad \varphi_{\text {int }}^{(* \lim )}=\max _{d V}\left[\varphi_{\text {int }}\left(F_{* \lim }, d V\right)\right] .
$$

Consequently, if we consider the tensor $\varphi_{i j}$ in each elementary volume $d V$ of a body, then in the general case, to describe a change in the value of acting stresses, as compared to the value of limiting stresses, we may introduce three relative damaging stresses $[2,4]$ :

a component one

$$
\Psi_{i j}=\left|\varphi_{i j} / \varphi_{\mathrm{m}}^{(* \lim )}\right|
$$

a principal one

$$
\psi_{i}=\left|\varphi_{i} / \varphi_{1}^{(* \lim )}\right|
$$

and an octahedral one

$$
\psi_{\text {int }}=\varphi_{\text {int }} / \varphi_{\text {int }}^{(* \lim )},
$$

where, generally speaking, $\psi_{i j}$, $\psi_{i}$, and $\psi_{\text {int }}$ have a probabilistic nature, since under the strength conditions both the acting stresses $\varphi_{i j}, \varphi_{i}, \varphi_{\text {int }}$ and their limiting values $\varphi_{\mathrm{m}}^{(* \lim )}, \varphi_{i}^{(* \lim )}, \varphi_{\mathrm{int}}^{(* \lim )}$ are random quantities with the corresponding distribution densities.

The conditions for limiting dangerous volumes with account for Eqs. (1) are written as

$$
\begin{gathered}
V_{i j}=\left\{d V / \varphi_{i j} \geq \varphi_{\mathrm{m}}^{(* \lim )}, d V \subset V_{\mathrm{k}}\right\}, \quad i, j=x, y, z, \quad \mathrm{~m}=\left\{\begin{array}{lll}
\mathrm{n} & \text { at } & i=j, \\
\tau & \text { at } & i \neq j,
\end{array}\right. \\
V_{i}=\left\{d V / \varphi_{i} \geq \varphi_{1}^{(* \lim )}, d V \subset V_{\mathrm{k}}\right\}, \quad i=1,2,3, \\
V_{\mathrm{int}}=\left\{d V / \varphi_{\mathrm{int}} \geq \varphi_{1}^{(* \lim )}, d V \subset V_{\mathrm{k}}\right\} .
\end{gathered}
$$

Finite-Element Modeling of damaged state. Since dangerous volumes can have an arbitrary and complex shape, their analytical determination from Eqs. (1)-(3) can be difficult; in such cases they are calculated by the Monte Carlo method [1-3]. In practical applications, of great value is the determination of the damaged state on the basis of the finite-element modeling of the stressed-strained state. 


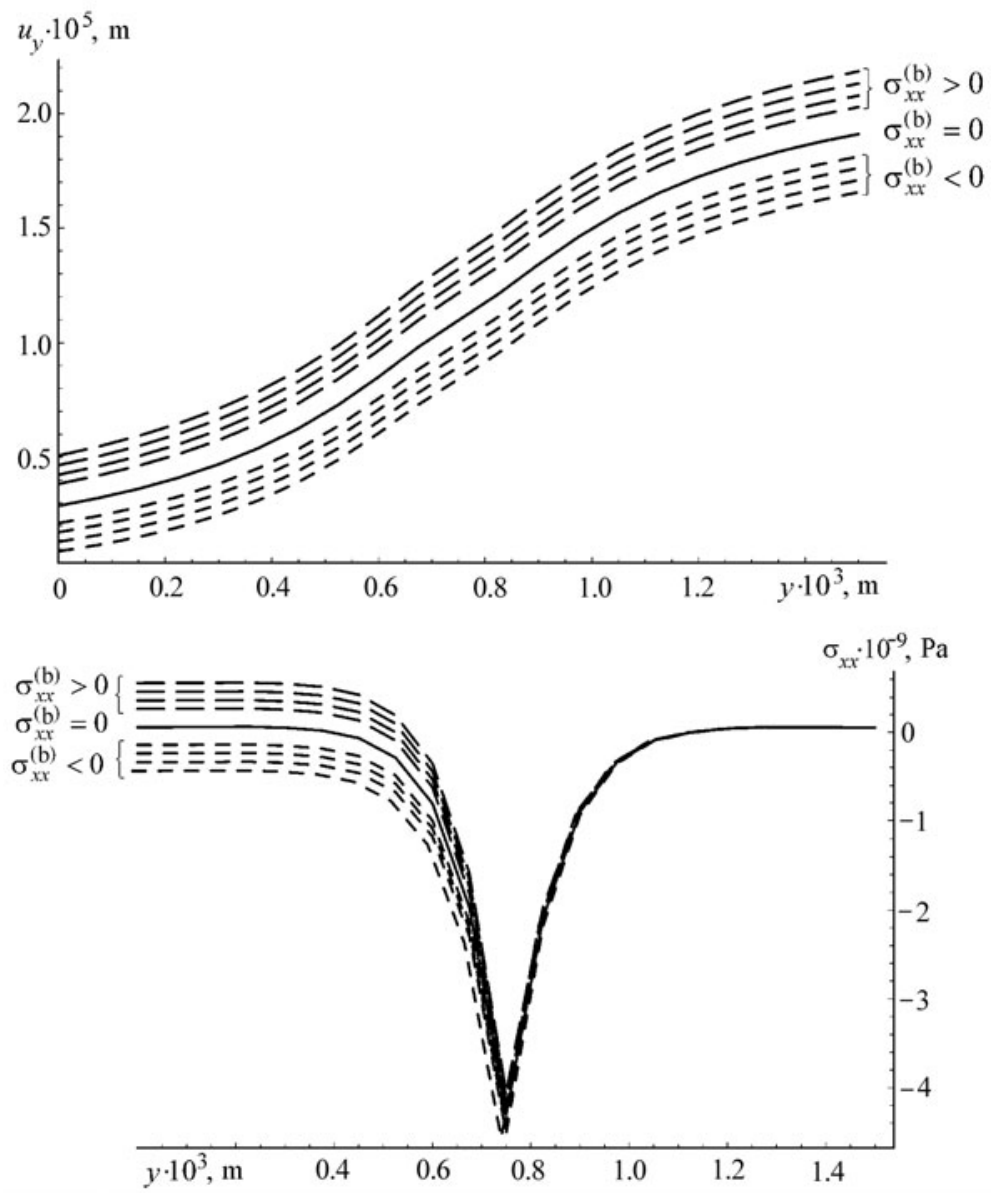

Fig. 2. Change in the displacements $u_{y}$ and stresses $\sigma_{x x}$ under the action of noncontact loading.

We consider modeling of the damaged state of a large number of finite elements in the vicinity of a contact using as an example computer modeling of the indenter/half-space system presented in Fig. 1. The principal curvature radii of the modeled indenter/elastic half-space system were as follows: $R_{11}=0.003 \mathrm{~m}, R_{12}=0.008 \mathrm{~m}, R_{21}=$ $\infty$, and $R_{12}=\infty$. The contact loading was equal to $F_{\mathrm{N}}=1200 \mathrm{~N}$, the properties of the material were determined by Young's moduli $E_{1}=E_{2}=2.01 \cdot 10^{11} \mathrm{~Pa}$, the Poisson coefficient was equal to $v=0.3$. For the selected loading and geometric characteristics of the system investigated, the values of its larger and smaller half-axes were equal to $a=$ $4.67 \cdot 10^{-4} \mathrm{~m}, b=2.44 \cdot 10^{-4} \mathrm{~m}(b / a=0.52)$. In the model shown in Fig. 1, the half-axis $a$ is codirectional with the coordinate axis $z$.

The half-space is also loaded by a uniformly distributed stretching or compressing normal stress in the direction $x$ : $\sigma_{x x}^{(b)}$ in such a way that in the vicinity of the contact the following relation holds [1-3]:

$$
\sigma_{x x}=\sigma_{x x}^{(\mathrm{c})}+\sigma_{x x}^{(\mathrm{b})}
$$

A contact loading $F_{\mathrm{N}}$ was applied to the upper plane of the indenter, and stresses $\sigma_{x x}^{(\mathrm{b})}$ varying in the ranges from -500 to $-200,0,200-500 \mathrm{MPa}$ were applied to the end face of a cube that models the half-space. The calculation was performed for one-fourth of the initial model with account for the symmetry conditions. The error of the results of the finite-element modeling was estimated with the aid of the equation [5]

$$
\varepsilon_{y y}=\frac{\sigma_{y y}-\sigma_{y y}^{\mathrm{A}}}{\sigma_{y y, \max }^{\mathrm{A}}} .
$$




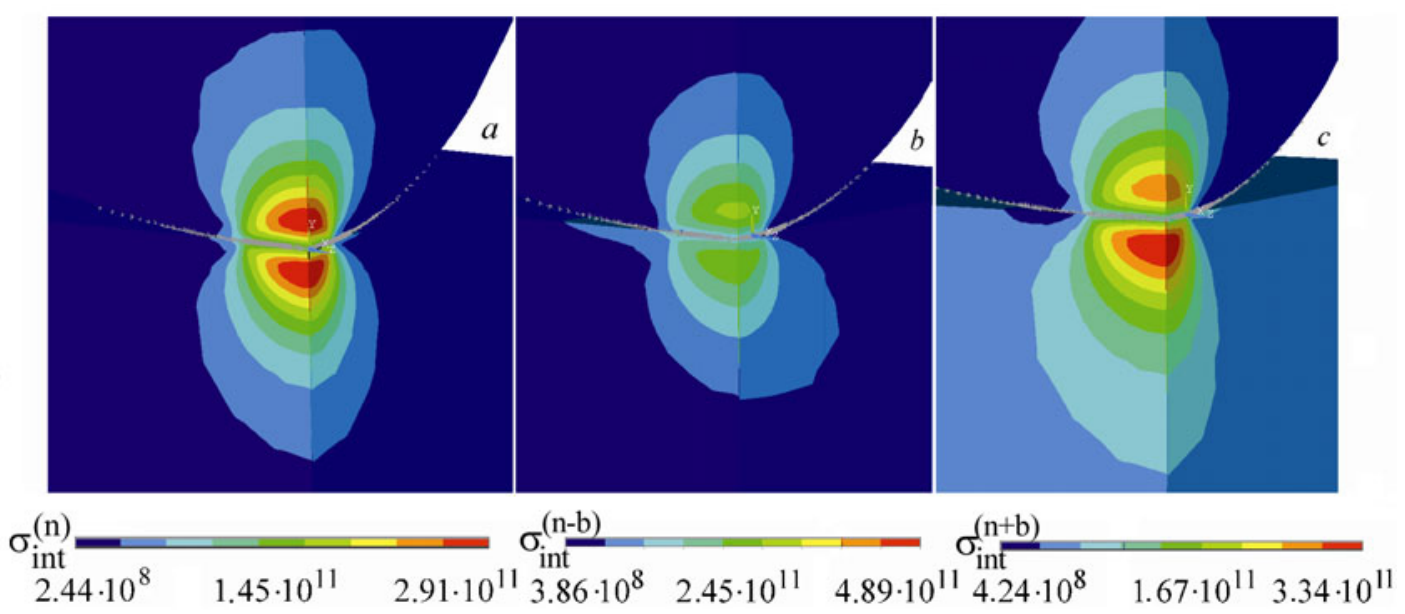

Fig. 3. Intensity of stresses in the case of contact interaction in the absence of noncontact loading (a) and under the loads $\sigma_{x x}^{(b)}=-500 \mathrm{MPa}$ (b) and $\sigma_{x x}^{(\mathrm{b})}=$ $500 \mathrm{MPa}(\mathrm{c})$.
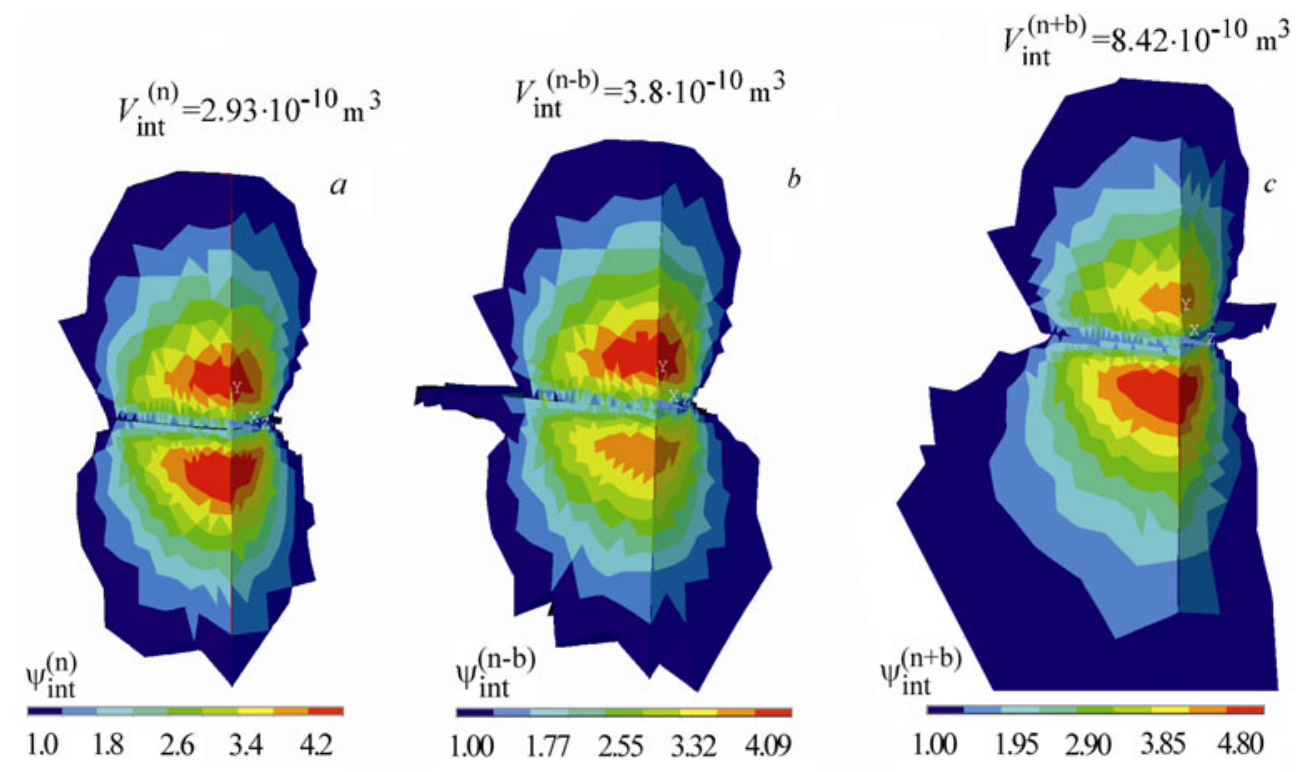

Fig. 4. Dangerous volumes and characteristics of the local damaged state in the absence of contact loading (a) and under the loads $\sigma_{x x}^{(b)}=-500 \mathrm{MPa}$ (b) and $\sigma_{x x}^{(b)}=500 \mathrm{MPa}(\mathrm{c})$.

The average value of the error along the $y$ axis was equal to $4.050 \cdot 10^{-3}$, and the maximum one to $1.744 \cdot 10^{-2}$, which is indicative of the good accuracy of the finite-element modeling.

The presence of a contact loading is responsible for the substantial change in the stressed-strained state of the vicinity of the contact calculated according to Eq. (4). Figure 2 shows the characteristic features of the change in displacements and normal stresses along the vertical axis $y$ passing through the contact center. It is seen from the figure that the region of contact descends because of transverse contraction under the action of stetching noncontact stresses and ascends under the action of compressing ones. Seen distinctly is also the change in the stresses $\sigma_{x x}$, which is explained by the application of a noncontact loading along the $x$ axis. Here, in the cube the compressing stresses $\sigma_{x x}$ change according to Eq. (1), and in the indenter the stresses $\sigma_{x x}^{(\mathrm{c})}$ are determined only by contact interaction.

An analysis of the damaged state of the system was carried out on the basis of a model of a deformed solid body with a dangerous volume for the lower boundary of the endurance limit scattering in the case of contact fatigue characterized, according to Eq. (1), by the value $p_{\mathrm{f}}=888 \mathrm{MPa}$. 
To realize the calculation of dangerous volumes, a program with the aid of the APDL language incorporated into ANSYS has been developed. The principle of operation of the program is as follows [6]. Carrying out the calculations for the $i$ th finite element yields the values of the average stresses and deformations. These values are carried over into the array, which is used in further calculations of the values of the damaged state as the ratio of the acting and limiting stresses in accordance with Eq. (2):

$$
\psi^{i}=\sigma_{\text {int }}^{i} / p_{\mathrm{f}}
$$

The volumes of the elements for which the following condition holds;

$$
\psi^{i} \geq 1
$$

are summed up to obtain the value of the dangerous volume $V_{\text {int }}$ for the entire computational model. On execution of the program, the visualization of one-fourth of the dangerous volumes with the distribution of damages in the contacting bodies was made.

Analysis of the distribution of the intensity of stresses (Fig. 3) and of corresponding damages of the indenter and half-space (Fig. 4) shows that the dangerous volume $V_{\text {int }}^{(\mathrm{n} \pm \mathrm{b})}$ calculated according to Eq. (3) and the damage $\psi_{\text {int }}^{(\mathrm{n} \pm \mathrm{b})}$ during the action of noncontact loading increase as compared to $V_{\text {int }}^{(\mathrm{n})}$ and $\psi_{\text {int }}^{(\mathrm{n})}$ in the case of a purely contact interaction on both compression and stretching due to the action of the contact force. In this case

$$
V_{\text {int }}^{(\mathrm{n})} \leq V_{\mathrm{int}}^{(\mathrm{n}-\mathrm{b})} \leq V_{\mathrm{int}}^{(\mathrm{n}+\mathrm{b})}
$$

Conclusions. The choice of the limiting criterion of the damaged state of solid bodies in the vicinity of their contact interaction and of equivalent stresses depends on the type of interaction: for sliding friction during which there are high shear stresses on the surfaces of the interacting bodies, it is worthwhile to estimate the damaged state of these bodies using $V_{1}$ or the component dangerous volume in the direction of friction. In the case of rolling friction where the friction coefficient is very small and the zone of intense cracking formation is located under the surface of the bodies, the estimation of their damaged state by $V_{\text {int }}$ can be more efficient.

\section{NOTATION}

$a$ and $b$, larger and smaller semi-axes of the contact ellipse; $E_{1}$ and $E_{2}$, Young's moduli; $F_{\mathrm{N}}$, contact loading; $F_{* \lim }$, limiting loading; $p_{\mathrm{f}}$, endurance limit during contact fatigue; $R_{i j}$, principal radii of curvature of the indenter/elastic half-space system; $d V$, elementary volume of a loaded body; $V_{\mathrm{k}}$, working volume of a body; $V_{\text {int }}$, dangerous volume of a body; $v$, Poisson coefficient; $\varepsilon_{y y}$, error of the results of finite element modeling; $\varepsilon_{i j}$ and $\sigma_{i j}$, stress and strain tensors; $\sigma_{y y}$, stress calculated by the finite element method; $\varphi_{i j}$, tensor of mechanical parameter; $\varphi_{i j}^{( \pm * \lim )}$, tensor of mechanical parameter under limiting loading; $\varphi_{1}^{(* \lim )}$, limiting principal value of the tensor $\varphi_{i j}^{( \pm * \lim )} ; \varphi_{\text {int }}^{(* \lim )}$, limiting intensity of the tensor $\varphi_{i j}^{( \pm * \lim )} ; \psi_{i j}, \psi_{i}$, and $\psi_{\text {int }}$, component, principal, and octahedral relative damaging stresses. Indices: $i$ and $j$ take the values of $x, y$, and $z$ to denote the corresponding components of the stress tensor; n, normal value of the parameter; $\tau$, tangential value of the parameter; $m$ takes the values of $n$ or $\tau$; $c$, the value of the parameter in the case of a purely contact interaction; $b$, the value of the parameter in the case of noncontact stretching-compression; ( $n$ - b), parameters at stresses caused by contact interaction and noncontact compression; $(n+b)$, parameters at stresses caused by contact interaction and noncontact stretching; $(n \pm b)$, parameters at stresses caused by contact interaction and noncontact stretching or compression; max, maximum; lim, limiting; 1, principal value of a parameter.

\section{REFERENCES}

1. L. A. Sosnovskii, Mechanics of Wear Fatigued Damage [in Russian], BelGUT, Gomel' (2007).

2. M. A. Zhuravkov and S. S. Shcherbakov, Analysis of the force system impairment with the aid of the model of a deformable solid body having a dangerous volume, Vestsi Nats. Akad. Navuk Belarusi, Ser. Fiz.-Tékh. Navuk, No. 4, 40-43 (2010). 
3. S. S. Shcherbakov, Contact problem. Analysis of dangerous volumes, in: Volume of Scientific papers "Problems of Computational Mechanics and of the Strength of Constructions," Issue 13, 279-285 (2009).

4. S. S. Shcherbakov, Stressed-strained state of the disk-cylinder system under contact interaction and deflection of a cylinder, Vestsi Nats. Akad. Navuk Belarusi, Ser. Fiz.-Tékh. Navuk, No. 3, 29-34 (2010).

5. K. Johnson, Mechanics of Contact Interaction [Russian translation], Mir, Moscow (1989).

6. I. N. Pavlovskii and S. S. Shcherbakov, Computer simulation of the damage of a tribologically reliable system with the aid of a model of a deformable solid body with a dangerous volume, in: Proc. 6th Int. Symp. on Tribological Reliability, October 25-November 1, 2010, Minsk (Belarus), BGU, Minsk (2010), Vol. 2, pp. 393398. 